\title{
Boundedness for a bilinear model sum operator on $\mathbb{R}^{n}$
}

by

ERIN Terwilleger (Storrs, CT)

\begin{abstract}
The purpose of this article is to obtain a multidimensional extension of Lacey and Thiele's result on the boundedness of a model sum which plays a crucial role in the boundedness of the bilinear Hilbert transform in one dimension. This proof is a simplification of the original proof of Lacey and Thiele modeled after the presentation of Bilyk and Grafakos.
\end{abstract}

1. Introduction. In the past decade much progress has been made in the theory of multilinear singular integral operators. Lacey and Thiele $[10,11]$ revived this area with their proof of boundedness for the bilinear Hilbert transforms. In one dimension, the bilinear Hilbert transforms in the direction $(\alpha, \beta)$ are given by

$$
H_{\alpha, \beta}\left(f_{1}, f_{2}\right)(x)=\text { p.v. } \int_{\mathbb{R}} f_{1}(x-\alpha t) f_{2}(x-\beta t) \frac{d t}{t}, \quad x \in \mathbb{R},
$$

where $f_{1}$ and $f_{2}$ are Schwartz functions. In their work, Lacey and Thiele reduced the boundedness of $H_{\alpha, \beta}$ to that of model sums of the form

$$
\sum_{\left(s_{1}, s_{2}, s_{3}\right) \in \mathbf{S}}\left|I_{s}\right|^{-1 / 2}\left\langle f_{1}, \phi_{s_{1}}\right\rangle\left\langle f_{2}, \phi_{s_{2}}\right\rangle \phi_{s_{3}},
$$

where $\mathbf{S}$ is a set of triples of tiles with the same time component and $\phi_{s_{j}}$ is a smooth bump adapted to a tile $s_{j}$ in an appropriate way. Then Lacey and Thiele employed a relatively straightforward, but technical, argument to obtain the $L^{p}$ boundedness of $H_{\alpha, \beta}$ for $p>2 / 3$ as a consequence of that for the operators in (1.1).

We became interested in how the theory evolves in higher dimensions. In particular, we are interested in understanding the boundedness properties of the bilinear Hilbert transform on $\mathbb{R}^{n}$,

2000 Mathematics Subject Classification: Primary 42B20; Secondary 46B70, 47B38.

Key words and phrases: multilinear operators in higher dimensions, bilinear Hilbert transform. 


$$
H_{v_{1}, v_{2}}(f, g)(x)=\text { p.v. } \int_{\mathbb{R}} f\left(x-t v_{1}\right) g\left(x-t v_{2}\right) \frac{d t}{t}, \quad x \in \mathbb{R}^{n},
$$

where $v_{1}$ and $v_{2}$ are nonzero vectors in $\mathbb{R}^{n}$ such that $v_{1} \neq v_{2}$. If one of $v_{1}$ and $v_{2}$ is zero, then the operator $H_{v_{1}, v_{2}}$ reduces to a product of a directional Hilbert transform and another function.

The above question is still elusive. The problem at present seems to be the passage from the model sum to the operator. In fact, it is not clear if this is even possible. The problem is there are too many free frequency parameters. However, motivated by the boundedness of the one-dimensional model sum, we are able to obtain restricted weak type bounds for the model sums in higher dimensions. Using bilinear interpolation between adjoint operators, (see Grafakos and Tao [6]), one is then able to obtain the $L^{p_{1}} \times L^{p_{2}} \rightarrow L^{p}$ boundedness of the aforementioned model sum when $1 / p_{1}+1 / p_{2}=1 / p$, $1<p_{1}, p_{2}<\infty$, and $p>2 / 3$.

Of course in $[10,11]$, the assumptions on the sums are dictated by the decomposition of the operators into averages of the model sums. In higher dimensions, it is not clear how this decomposition will work. In this sense the assumptions we make on our model sums are somewhat forced, although they are similar to those in $[10,11]$. However, the model sums are believed to play an important role in obtaining bounds for the operators in (1.2).

A feature of our proof is that, even in dimension $n=1$, it provides a simplification of the original proof given by Lacey and Thiele in [11]. Bilyk and Grafakos $[1,2]$ use a similar simplification to obtain distributional estimates for the bilinear Hilbert transform in an extended range of exponents. We use the same idea as in the aforementioned two papers of estimating the operator on and off an "exceptional" set. The simplification comes in the form of an improved energy estimate similar to one used by Grafakos, Tao, and Terwilleger [7] to prove $L^{p}$ bounds for a higher dimensional maximal dyadic operator, which in one dimension is related to the Carleson operator. This idea can also be found in the paper of Muscalu, Tao, and Thiele [13] on multilinear operators. In addition, the proof uses a selection process inspired by the one given by Lacey [9] in the case $L^{2} \times L^{2} \rightarrow L^{1, \infty}$.

2. Multidimensional extension of the model sum. We begin by setting up the notation and assumptions used in our model. A dyadic cube $I \subset \mathbb{R}^{n}$ is of the form

$$
I=\prod_{i=1}^{n} I^{(i)}=\prod_{i=1}^{n}\left[m_{i} 2^{k},\left(m_{i}+1\right) 2^{k}\right),
$$

where $k$ and $m_{i}$ are integers for all $i=1, \ldots, n$. The $n$-dimensional volume is given by $|I|=2^{n k}$. Let $c(I)=\left(c\left(I^{(1)}\right), \ldots, c\left(I^{(n)}\right)\right)$ denote the center 
of $I$, and for $a>0, a I$ will denote the cube with the same center as $I$ and whose volume is $a^{n}|I|$. We will be considering functions of time in $n$ dimensional space whose Fourier transforms are functions of frequency in $n$-dimensional space. These functions will be adapted to a "rectangle" in the $2 n$-dimensional time-frequency plane. A dyadic rectangle $s$ is the tensor product of a dyadic cube, $I_{s}$, from the time plane and a dyadic cube, $\omega_{s}$, from the frequency plane. A dyadic rectangle $s=I_{s} \times \omega_{s}$ of volume one is called a tile. We will denote the set of generic rectangles in $2 n$-dimensional space by $\mathcal{R}$.

Since we will be working with three functions we need to define the notion of a tri-tile. A tri-tile is a rectangle $s=I_{s} \times \omega_{s}$ together with three tiles $s_{1}, s_{2}, s_{3}$ with the following properties:

(a) $I_{s}=I_{s_{j}}$ for $j=1,2,3$.

(b) $\mathcal{J}=\bigcup_{s \in \mathcal{R}}\left(\omega_{s} \cup \omega_{s_{1}} \cup \omega_{s_{2}} \cup \omega_{s_{3}}\right)$ is a grid.

(c) $\omega_{s}$ is the convex hull of $\omega_{s_{1}}, \omega_{s_{2}}$, and $\omega_{s_{3}}$.

(d) For each $i=1, \ldots, n, \xi_{1}^{(i)}<\xi_{2}^{(i)}<\xi_{3}^{(i)}$ for all $\xi_{j} \in \omega_{s_{j}}$ and $j=1,2,3$, and $\operatorname{dist}\left(\omega_{s_{j}}^{(i)}, \omega_{s_{j^{\prime}}}^{(i)}\right)=c_{j, j^{\prime}}\left|I_{s}\right|^{-1 / n}$ for $j \neq j^{\prime}$ and a fixed constant $c_{j, j^{\prime}}$.

Property (d) is a separation condition motivated by the one-dimensional case in which it can be shown that the model sums for the bilinear Hilbert transform satisfy such a condition [10]. For our analysis, we also need the following property which essentially follows from a separation of scales:

(2.1) For two fixed tri-tiles $s$ and $s^{\prime}$, if there exists an $i \in\{1,2,3\}$ such that $\omega_{s_{i}} \subsetneq \omega_{s_{j}^{\prime}}$ for some fixed $j=1,2,3$, then $\omega_{s} \subset \omega_{s_{j}^{\prime}}$.

We will denote the set of tri-tiles with property (2.1) by $\mathbf{D}$.

We fix a Schwartz function $\phi$ such that $\widehat{\phi}$ is real, nonnegative, supported in the cube $[-1 / 10,1 / 10]^{n}$ and equal to 1 on the cube $[-9 / 100,9 / 100]^{n}$. For a tri-tile $s \in \mathbf{D}$ and $x \in \mathbb{R}^{n}$ we define

$$
\phi_{s_{j}}(x)=\left|I_{s}\right|^{-1 / 2} \phi\left(\frac{x-c\left(I_{s}\right)}{\left|I_{s}\right|^{1 / n}}\right) e^{2 \pi i c\left(\omega_{s_{j}}\right) \cdot x}, \quad j=1,2,3 .
$$

Using the following definition of the Fourier transform:

$$
\widehat{f}(\eta)=\int_{\mathbb{R}^{n}} f(x) e^{-2 \pi i x \cdot \eta} d x
$$

one can easily see that

$$
\widehat{\phi}_{s_{j}}(\eta)=\left|\omega_{s_{j}}\right|^{-1 / 2} \widehat{\phi}\left(\frac{\eta-c\left(\omega_{s_{j}}\right)}{\left|\omega_{s_{j}}\right|^{1 / n}}\right) e^{2 \pi i c\left(I_{s}\right) \cdot\left(c\left(\omega_{s_{j}}\right)-\eta\right)}, \quad j=1,2,3 .
$$

Definition (2.2) tells us that for each $s_{j}$ the function $\phi_{s_{j}}$ is well localized in space with most of its mass in $I_{s}$ while (2.3) tells us that $\widehat{\phi}_{s_{j}}$ is supported in $\omega_{s_{j}}$. Note also that the $\phi_{s_{j}}$ have the same $L^{2}\left(\mathbb{R}^{n}\right)$ norm. 
Let

$$
\langle f, g\rangle=\int_{\mathbb{R}^{n}} f(x) \overline{g(x)} d x
$$

be the usual complex inner product. For a finite set $\mathbf{S}$ of tri-tiles, we define the higher dimensional bilinear sum

$$
H_{\mathbf{S}}\left(f_{1}, f_{2}\right):=\sum_{s \in \mathbf{S}}\left|I_{s}\right|^{-1 / 2}\left\langle f_{1}, \phi_{s_{1}}\right\rangle\left\langle f_{2}, \phi_{s_{2}}\right\rangle \phi_{s_{3}},
$$

where $f_{1}$ and $f_{2}$ are initially taken to be Schwartz functions. Our main result is the following theorem.

TheOrem 1. Let $\mathbf{S}$ be a finite collection of tri-tiles in $\mathbf{D}$ and $f_{1}$ and $f_{2}$ be Schwartz functions. Then for $1 / p_{1}+1 / p_{2}=1 / p, 1<p_{1}, p_{2}<\infty$, and $p>2 / 3$,

$$
\left\|H_{\mathbf{S}}\left(f_{1}, f_{2}\right)\right\|_{L^{p}\left(\mathbb{R}^{n}\right)} \leq C\left\|f_{1}\right\|_{L^{p_{1}\left(\mathbb{R}^{n}\right)}}\left\|f_{2}\right\|_{L^{p_{2}\left(\mathbb{R}^{n}\right)}}
$$

where $C$ is independent of the collection $\mathbf{S}$ and the functions $f_{1}$ and $f_{2}$.

Theorem 1 will follow from a restricted weak type estimate using interpolation and duality. In particular we will prove the following theorem.

Theorem 2. Let $r_{1}$ and $r_{2}$ be such that $r_{1}, r_{2}>1$ and $1 / r_{1}+1 / r_{2}=3 / 2$. Let $1 / p_{1}=1 / r_{1}-\varepsilon, 1 / p_{2}=1 / r_{2}-\varepsilon$, and $1 / p=3 / 2-2 \varepsilon$ where $\varepsilon$ is a small positive number. Given $n$-dimensional sets $F_{1}, F_{2}$, and $E$ of finite measure, there exists a set $E^{\prime} \subset E$ with $\left|E^{\prime}\right| \geq \frac{1}{2}|E|$ such that

$$
\int_{E^{\prime}} H_{\mathbf{S}}\left(\chi_{F_{1}}, \chi_{F_{2}}\right)(x) d x \lesssim \frac{\left|F_{1}\right|^{1 / p_{1}}\left|F_{2}\right|^{1 / p_{2}}}{|E|^{1 / p-1}} .
$$

Assuming Theorem 2 holds, $H_{\mathbf{S}}$ is bounded for $\left|1 / p_{1}-1 / p_{2}\right|<1 / 2$ and $2 / 3<p<2$ by interpolation. See, for example, the general interpolation theorem for multilinear operators of Grafakos and Tao [6]. Then one is able to recover Theorem 1 for the full range of exponents from duality.

To prove Theorem 2, we first determine the set $E^{\prime}$. Given $n$-dimensional sets $F_{1}, F_{2}$, and $E$ of finite measure, define

$$
\begin{aligned}
\Omega= & \left\{x: M\left(\chi_{F_{1}}\right)>12^{n} \min \left(1,\left|F_{1}\right| /|E|\right)\right\} \\
& \cup\left\{x: M\left(\chi_{F_{2}}\right)>12^{n} \min \left(1,\left|F_{2}\right| /|E|\right)\right\},
\end{aligned}
$$

where $M$ is the usual Hardy-Littlewood maximal function. Set $E^{\prime}:=E \backslash \Omega$. Since $M$ is of weak type $(1,1)$ with constant at most $3^{n}$, we see that $|\Omega|<$ $\frac{1}{2}|E|$, and hence $\left|E^{\prime}\right| \geq \frac{1}{2}|E|$. Note that since $M\left(\chi_{F_{i}}\right) \leq 1, \Omega=\emptyset$ if $|E| \leq$ $\min \left(\left|F_{1}\right|,\left|F_{2}\right|\right)$. We will need the following estimates. 
Proposition 1. Let $\mathbf{S}$ be a finite collection of tri-tiles in $\mathbf{D}$. Then $\int_{E^{\prime}} H_{\left\{s \in \mathbf{S}: I_{s} \subseteq \Omega\right\}}\left(\chi_{F_{1}}, \chi_{F_{2}}\right)(x) d x \leq C \min \left(\left|F_{1}\right|^{1 / 2},\left|F_{2}\right|^{1 / 2}\right) \frac{\left|F_{1}\right|^{1 / 2}\left|F_{2}\right|^{1 / 2}}{|E|^{1 / 2}}$, where $C$ is independent of the collection $\mathbf{S}$.

Proposition 2. Let $\mathbf{S}$ be a finite collection of tri-tiles in $\mathbf{D}$. Then

$$
\begin{aligned}
\int_{E^{\prime}} H_{\left\{s \in \mathbf{S}: I_{s} \nsubseteq \Omega\right\}} & \left(\chi_{F_{1}}, \chi_{F_{2}}\right)(x) d x \\
\leq & C \min \left(\left|F_{1}\right|^{1 / 2},\left|F_{2}\right|^{1 / 2}\right) \min \left(\frac{\left|F_{1}\right|^{1 / 2}\left|F_{2}\right|^{1 / 2}}{|E|^{1 / 2}},|E|^{1 / 2}\right) \\
& \times\left(1+\left|\log \frac{\left|F_{1}\right|}{|E|}\right|+\left|\log \frac{\left|F_{2}\right|}{|E|}\right|\right),
\end{aligned}
$$

where $C$ is independent of the collection $\mathbf{S}$.

We now prove Theorem 2, and hence Theorem 1, assuming that Propositions 1 and 2 hold. In the next section we prove Proposition 1, which is the more straightforward estimate. Then in Section 4 we prove Proposition 2 which requires a proper organization of the tiles that contribute to the sum.

Proof of Theorem 2. First notice that Propositions 1 and 2 together imply that

$$
\begin{aligned}
& \int_{E^{\prime}} H_{\mathbf{S}}\left(\chi_{F_{1}}, \chi_{F_{2}}\right)(x) d x \\
\lesssim & \left.\min \left(\left|F_{1}\right|^{1 / 2},\left|F_{2}\right|^{1 / 2}\right) \frac{\left|F_{1}\right|^{1 / 2}\left|F_{2}\right|^{1 / 2}}{|E|^{1 / 2}}\left(1+\mid \log \frac{\left|F_{1}\right|}{|E|}\right)\right)\left(1+\left|\log \frac{\left|F_{2}\right|}{|E|}\right|\right) .
\end{aligned}
$$

We begin with the case $|E| \geq\left|F_{1}\right|,\left|F_{2}\right|$. Notice that by the choice of $r_{1}$ and $r_{2},(2.5)$ implies that

$$
\int_{E^{\prime}} H_{\mathbf{S}}\left(\chi_{F_{1}}, \chi_{F_{2}}\right)(x) d x \lesssim \frac{\left|F_{1}\right|^{1 / r_{1}}\left|F_{2}\right|^{1 / r_{2}}}{|E|^{1 / 2}}\left(1+\log \frac{|E|}{\left|F_{1}\right|}\right)\left(1+\log \frac{|E|}{\left|F_{2}\right|}\right) .
$$

Thus (2.4) follows as $1+\log a \lesssim a^{\varepsilon}$ for $a \geq 1$.

If $|E| \leq\left|F_{1}\right|,\left|F_{2}\right|$, then as noted above, $\Omega$ is the empty set, and so we need only Proposition 2 . In this case the inequality becomes

$$
\begin{aligned}
& \int_{E^{\prime}} H_{\mathbf{S}}\left(\chi_{F_{1}}, \chi_{F_{2}}\right)(x) d x \\
& \lesssim \min \left(\left|F_{1}\right|^{1 / 2},\left|F_{2}\right|^{1 / 2}\right)|E|^{1 / 2}\left(1+\log \frac{\left|F_{1}\right|}{|E|}\right)\left(1+\log \frac{\left|F_{2}\right|}{|E|}\right) \\
& \lesssim\left|F_{1}\right|^{1 / r_{1}-1 / 2}\left|F_{2}\right|^{1 / r_{2}-1 / 2}|E|^{1 / 2}\left(\frac{\left|F_{1}\right|}{|E|}\right)^{1 / 2-\varepsilon}\left(\frac{\left|F_{2}\right|}{|E|}\right)^{1 / 2-\varepsilon}
\end{aligned}
$$

which is just (2.4). 
Finally, we consider the case $\left|F_{1}\right| \leq|E| \leq\left|F_{2}\right|$. Here we need to be more clever in our exponents. Fix $\delta$ small enough so that $\alpha:=1 / r_{1}-\varepsilon+\delta \leq 1$ and $\beta:=1 / r_{2}-\delta+\varepsilon \leq 1$. Note that $\alpha+\beta=3 / 2$. Now in (2.5) we replace the exponent for $\left|F_{1}\right|$ with $\alpha+\beta-1$ and use the fact that $\left|F_{1}\right| \leq\left|F_{2}\right|$ to obtain

$$
\begin{aligned}
\int_{E^{\prime}} H_{\mathbf{S}}\left(\chi_{F_{1}}, \chi_{F_{2}}\right)(x) d x & \lesssim \frac{\left|F_{1}\right|^{\alpha}\left|F_{2}\right|^{\beta}}{|E|^{1 / 2}}\left(1+\log \frac{|E|}{\left|F_{1}\right|}\right)\left(1+\log \frac{\left|F_{2}\right|}{|E|}\right) \\
& \lesssim \frac{\left|F_{1}\right|^{\alpha}\left|F_{2}\right|^{\beta}}{|E|^{1 / 2}}\left(\frac{|E|}{\left|F_{1}\right|}\right)^{\delta}\left(\frac{\left|F_{2}\right|}{|E|}\right)^{\delta-2 \varepsilon},
\end{aligned}
$$

which is again just (2.4).

3. Proof of Proposition 1. This proof is modeled after similar ones in Bilyk and Grafakos [1] and Grafakos, Tao, and Terwilleger [7] whose original inspiration came from Lacey and Thiele [11]. We will prove this in the case $\left|F_{1}\right| \leq\left|F_{2}\right|$, as the operator is symmetric in $F_{1}$ and $F_{2}$.

For a dyadic cube $J \subseteq \Omega$ and $\gamma$ a large integer to be chosen later, we set

$$
\mathbf{S}_{J}:=\left\{s \in \mathbf{S}: I_{s}=J\right\}
$$

and

$$
\psi_{J}(x):=\left(1+\frac{|x-c(J)|}{|J|^{1 / n}}\right)^{-\gamma n} .
$$

Notice that since $\phi$ is a Schwartz function,

$$
\left|\phi_{s_{i}}(x)\right| \lesssim|J|^{-1 / 2} \psi_{J}(x)
$$

for $i=1,2,3$ and $s \in \mathbf{S}_{J}$.

We also need the following variants of Bessel's inequality. For $i=1,2,3$, and functions $\alpha_{s}: \mathbf{S}_{J} \rightarrow \mathbb{R}$,

$$
\begin{aligned}
\left\|\sum_{s \in \mathbf{S}_{J}} \alpha_{s} \phi_{s_{i}} \psi_{J}^{-1}\right\|_{2} & \lesssim\left\|\left(\alpha_{s}\right)\right\|_{\ell^{2}\left(\mathbf{S}_{J}\right)}, \\
\left\|\left(\left\langle f, \phi_{s_{i}}\right\rangle\right)\right\|_{\ell^{2}\left(\mathbf{S}_{J}\right)} & \lesssim\left\|f \psi_{J}\right\|_{2} .
\end{aligned}
$$

Since (3.3) is the dual statement to (3.2), we just show (3.2). First note that we have the almost orthogonality condition

$$
\begin{aligned}
\left|\left\langle\phi_{s_{i}} \psi_{J}^{-1}, \phi_{s_{i}^{\prime}} \psi_{J}^{-1}\right\rangle\right| & =\left|\left(|\phi(x)|^{2}(1+|x|)^{\gamma n}\right)^{\wedge}\left(\left[c\left(\omega_{s_{i}}\right)-c\left(\omega_{s_{i}^{\prime}}\right)\right]|J|^{1 / n}\right)\right| \\
& \lesssim\left(1+\left|c\left(\omega_{s_{i}}\right)-c\left(\omega_{s_{i}^{\prime}}\right)\right||J|^{1 / n}\right)^{-\gamma n} .
\end{aligned}
$$

In light of the properties of the tri-tiles and since $I_{s}=J$ is fixed for each $s$, $c\left(\omega_{s_{i}}\right)|J|^{1 / n}$ is a vector in $\mathbb{Z}^{n}$. Therefore we can index the elements $s \in \mathbf{S}_{J}$ 
by $\mathbb{Z}^{n}$. Taking the above facts into account, we have

$$
\begin{aligned}
\left\|\sum_{s \in \mathbf{S}_{J}} \alpha_{s} \phi_{s_{i}} \psi_{J}^{-1}\right\|_{2} & \leq \sum_{s, s^{\prime} \in \mathbf{S}_{J}}\left|\alpha_{s}\right|\left|\alpha_{s^{\prime}}\right|\left|\left\langle\phi_{s_{i}} \psi_{J}^{-1}, \phi_{s_{i}^{\prime}} \psi_{J}^{-1}\right\rangle\right| \\
& \lesssim \sum_{k, m \in \mathbb{Z}^{n}}\left|\alpha_{s_{k}}\right|\left|\alpha_{s_{m}}\right|(1+|k-m|)^{-\gamma n} \\
& \lesssim \sum_{k \in \mathbb{Z}^{n}}\left|\alpha_{s_{k}}\right|^{2} \sum_{m \in \mathbb{Z}^{n}}(1+|k-m|)^{-\gamma n} \lesssim\left\|\left(\alpha_{s}\right)\right\|_{\ell^{2}\left(\mathbf{S}_{J}\right)} .
\end{aligned}
$$

We set $M_{2}:=M\left(f^{2}\right)^{1 / 2}$.

Lemma 1. For any $A>1$, we have

$$
\left\|H_{\mathbf{S}_{J}}\left(\chi_{F_{1}}, \chi_{F_{2}}\right)\right\|_{L^{1}\left((A J)^{\mathrm{c}}\right)} \lesssim A^{-\gamma}|J| \inf _{x \in J} M\left(\chi_{F_{1}}\right)(x) \inf _{x \in J} M_{2}\left(\chi_{F_{2}}\right)(x) .
$$

Proof. Multiplying and dividing by $\psi_{J}$ we get

$$
\begin{aligned}
&\left\|H_{\mathbf{S}_{J}}\left(\chi_{F_{1}}, \chi_{F_{2}}\right)\right\|_{L^{1}\left((A J)^{\mathrm{c}}\right)} \leq\left\|\psi_{J}\right\|_{L^{2}\left((A J)^{\mathrm{c}}\right)}\left\|H_{\mathbf{S}_{J}}\left(\chi_{F_{1}}, \chi_{F_{2}}\right) \psi_{J}^{-1}\right\|_{L^{2}} \\
& \lesssim A^{-\gamma}|J|^{1 / 2}\left\|\sum_{s \in \mathbf{S}_{J}}|J|^{-1 / 2}\left\langle\chi_{F_{1}}, \phi_{s_{1}}\right\rangle\left\langle\chi_{F_{2}}, \phi_{s_{2}}\right\rangle \phi_{s_{3}} \psi_{J}^{-1}\right\|_{L^{2}} \\
& \lesssim A^{-\gamma}\left\|\left\langle\chi_{F_{1}}, \phi_{s_{1}}\right\rangle\left\langle\chi_{F_{2}}, \phi_{s_{2}}\right\rangle\right\|_{\ell^{2}\left(\mathbf{S}_{J}\right)} \quad \text { by }(3.2) \\
& \lesssim A^{-\gamma}\left\|\left\langle\chi_{F_{1}}, \phi_{s_{1}}\right\rangle\right\|_{\ell^{\infty}\left(\mathbf{S}_{J}\right)}\left\|\left\langle\chi_{F_{2}}, \phi_{s_{2}}\right\rangle\right\|_{\ell^{2}\left(\mathbf{S}_{J}\right)} \\
& \lesssim A^{-\gamma}|J|^{-1 / 2}\left\|\chi_{F_{1}} \psi_{J}\right\|_{L^{1}}\left\|\chi_{F_{2}} \psi_{J}\right\|_{L^{2}} \quad \text { by }(3.1) \text { and }(3.3) \\
& \lesssim A^{-\gamma}|J| \inf _{x \in J} M\left(\chi_{F_{1}}\right)(x) \inf _{x \in J} M_{2}\left(\chi_{F_{2}}\right)(x) \text {. }
\end{aligned}
$$

To utilize Lemma 1, we must organize the dyadic cubes $J \subseteq \Omega$ according to how they are imbedded in $\Omega$. Set

$$
\mathcal{F}_{k}:=\left\{J: 2^{k} J \subseteq \Omega, 2^{k+1} \nsubseteq \Omega\right\} .
$$

Letting $\mathcal{F}_{k}^{*}$ denote the dyadic cubes in $\mathcal{F}_{k}$ maximal with respect to inclusion, a critical observation is that

$$
\sum_{J \in \mathcal{F}_{k}}|J|=\sum_{J \in \mathcal{F}_{k}^{*}} \sum_{\substack{J^{\prime} \in \mathcal{F}_{k} \\ J^{\prime} \subseteq J}}\left|J^{\prime}\right| \leq 2^{n} \sum_{J \in \mathcal{F}_{k}^{*}}|J| \leq 2^{n}|\Omega| \leq 2^{n-1}|E| .
$$

This can be seen by noting that if $J^{\prime} \subseteq J$, then $J^{\prime}$ must share a boundary with $J$, otherwise $2 J^{\prime} \subseteq J$, which puts $J^{\prime}$ in $\mathcal{F}_{k+1}$. For $J^{\prime}$ of a fixed side length $2^{m}$ where $2^{m} \leq|J|^{1 / n}$, the measure of all such cubes is at most $2^{m} 2^{n}|J|^{(n-1) / n}$, which can be summed over $m \leq \log _{2}|J|^{1 / n}$. Finally, we use the fact that the elements of $\mathcal{F}_{k}^{*}$ are pairwise disjoint. 
Recalling that $E^{\prime} \subseteq \Omega^{\mathrm{c}} \subseteq\left(2^{k} J\right)^{\mathrm{c}}$ for each $k$ and applying Lemma 1, we have

$$
\begin{aligned}
\left|\int_{E^{\prime}} H_{\left\{s \in \mathbf{S}: I_{s} \subseteq \Omega\right\}}\left(\chi_{F_{1}}, \chi_{F_{2}}\right)(x) d x\right| \leq \sum_{k=0}^{\infty} \sum_{J \in \mathcal{F}_{k}}\left\|H_{\mathbf{S}_{J}}\left(\chi_{F_{1}}, \chi_{F_{2}}\right)\right\|_{L^{1}\left(\left(2^{k} J\right)^{\mathrm{c}}\right)} \\
\quad \lesssim \sum_{k=0}^{\infty} \sum_{J \in \mathcal{F}_{k}} 2^{-\gamma k}|J| \inf _{x \in J} M\left(\chi_{F_{1}}\right)(x) \inf _{x \in J} M_{2}\left(\chi_{F_{2}}\right)(x) \\
\lesssim \sum_{k=0}^{\infty} \sum_{J \in \mathcal{F}_{k}} 2^{-\gamma k} C^{2 k+2}|J| \inf _{x \in 2^{k+1} J} M\left(\chi_{F_{1}}\right)(x) \inf _{x \in 2^{k+1} J} M_{2}\left(\chi_{F_{2}}\right)(x) .
\end{aligned}
$$

Since $2^{k+1} J$ meets $\Omega^{\mathrm{c}}$, by definition we can bound the above by a constant multiple of

$$
\sum_{k=0}^{\infty} 2^{-\gamma k} C^{2 k+2} \sum_{J \in \mathcal{F}_{k}}|J| \frac{\left|F_{1}\right|}{|E|}\left(\frac{\left|F_{2}\right|}{|E|}\right)^{1 / 2} \lesssim\left|F_{1}\right|\left|F_{2}\right|^{1 / 2}|E|^{-1 / 2},
$$

where the last inequality follows from (3.4) and by choosing the constant $\gamma$ large enough so that $C^{2} 2^{-\gamma}<1$.

4. Proof of Proposition 2. We now set up some tools that will allow us to organize the large sums over tri-tiles in the statement of Proposition 2. Define a partial order $<$ on the set of tri-tiles $\mathbf{D}$ by setting

$$
s<s^{\prime} \Leftrightarrow I_{s} \subseteq I_{s^{\prime}} \text { and } \omega_{s^{\prime}} \subseteq \omega_{s} .
$$

We have the property that if two tri-tiles $s, s^{\prime} \in \mathbf{D}$ intersect, then either $s<s^{\prime}$ or $s^{\prime}<s$. To see this, first observe that dyadic cubes have the property that if two of them intersect, then one is contained in the other. If $s$ and $s^{\prime}$ intersect, then they intersect in both the time and frequency components, i.e. $I_{s} \cap I_{s^{\prime}} \neq \emptyset, \omega_{s} \cap \omega_{s^{\prime}} \neq \emptyset$. Without loss of generality, if we assume $\left|I_{s}\right| \leq\left|I_{s^{\prime}}\right|$, then $I_{s} \subseteq I_{s^{\prime}}$. Now, recall that $\omega_{s^{\prime}}$ and $\omega_{s}$ are not dyadic cubes but rather the closed convex hull of three dyadic cubes which are separated in each coordinate. By property (2.1) of the tri-tiles, if there exists an $i \in\{1,2,3\}$ such that $\omega_{s_{i}^{\prime}} \varsubsetneqq \omega_{s_{j}}$ for some fixed $j=1,2,3$, then $\omega_{s^{\prime}} \subseteq \omega_{s_{j}}$. On the other hand, if $\omega_{s_{i}^{\prime}} \nsubseteq \omega_{s_{j}}$ for all $i, j=1,2,3$, then $\omega_{s^{\prime}}$ must be contained in one of the spaces between the dyadic cubes $\omega_{s_{j}}$. In either case, we have $\omega_{s^{\prime}} \subseteq \omega_{s}$, which gives $s<s^{\prime}$. A consequence of this property is that for a finite set of tri-tiles, all maximal elements under $<$ must be disjoint sets.

A finite set $\mathbf{T}$ of tri-tiles is called a tree if there exists a tri-tile $t \in \mathbf{D}$ such that $s<t$ for all $s \in \mathbf{T}$. We call $t$ the top of the tree $\mathbf{T}$ and denote it by $t=I_{\mathbf{T}} \times \omega_{\mathbf{T}}$. The tiles composing the top of the tree will be denoted by $t_{j}=I_{\mathbf{T}} \times \omega_{\mathbf{T} j}$ for $j=1,2,3$. Note that the top is not necessarily an element 
of the tree. Another useful observation is that any finite set $\mathbf{S}$ of tri-tiles can be written as a union of trees by considering all maximal elements of $\mathbf{S}$ under $<$. Then a nonmaximal element $s \in \mathbf{S}$ must be less than, in the partial order, some maximal element $t \in \mathbf{S}$, which places $s$ in the tree with top $t$.

We say a tree $\mathbf{T}$ is of type $j=1,2,3$, or a $j$-tree, if

$$
\omega_{\mathbf{T}} \cap \omega_{s_{j}}=\emptyset \quad \text { for all } s \in \mathbf{T} .
$$

By the facts noted above, if $s<t$, where $t$ is the top of any tree $\mathbf{T}$, then either there is one $i \in\{1,2,3\}$ such that $\omega_{\mathbf{T}} \subseteq \omega_{s_{i}}$, or $\omega_{\mathbf{T}} \cap \omega_{s_{i}}=\emptyset$ for all $i=1,2,3$. If we set $\mathbf{T}_{0}:=\left\{s \in \mathbf{T}: \omega_{\mathbf{T}} \cap \omega_{s_{i}}=\emptyset\right.$ for all $\left.i=1,2,3\right\}$ and $\mathbf{T}_{i}:=\left\{s \in \mathbf{T}: \omega_{\mathbf{T}} \cap \omega_{s_{i}} \neq \emptyset\right\}$ for $i=1,2,3$, then $\mathbf{T}=\bigcup_{i=0}^{3} \mathbf{T}_{i}$. Also $\mathbf{T}_{0}$ is a tree of type 1,2 and 3 , while $\mathbf{T}_{i}$ is a tree of type $j \neq i$. By throwing the tri-tiles in $\mathbf{T}_{0}$ into one of the other sets $\mathbf{T}_{i}$, we see that any tree $\mathbf{T}$ can be written as a union of at most three pairwise disjoint subtrees which are $j$-trees for at least two choices of $j$.

Given any finite set $\mathbf{S}$ of tri-tiles, we want to find a suitable decomposition into sets on which we can apply estimates and obtain geometric series. This idea can be found in Lacey [9]. To this end, for a function $f \in L^{2}$ we define the $k$-energy of a finite set $\mathbf{S}$ of tri-tiles by

$$
\mathcal{E}_{k}(\mathbf{S}, f)=\frac{1}{\|f\|_{2}} \sup _{\substack{\mathbf{T} \subseteq \mathbf{S} \\ \mathbf{T} \text { of type } k}}\left(\left|I_{\mathbf{T}}\right|^{-1} \sum_{s \in \mathbf{T}}\left|\left\langle f, \phi_{s_{k}}\right\rangle\right|^{2}\right)^{1 / 2} .
$$

Notice that for the tri-tiles in a $k$-tree, the $\omega_{s_{k}}$ must be pairwise disjoint, and thus the Fourier supports of the functions $\phi_{s_{k}}$ are pairwise disjoint.

Lemma 2. Let $\mathbf{S}$ be a finite set of tri-tiles and $f$ a function in $L^{2}$. Then there exists a constant $C_{1}$ and a subset $\mathbf{S}_{1}$ of $\mathbf{S}$ such that $\mathbf{S}_{1}$ is a union of trees $\mathbf{T}_{l}$ and

$$
\mathcal{E}_{k}\left(\mathbf{S} \backslash \mathbf{S}_{1}, f\right) \leq \frac{1}{2} \mathcal{E}_{k}(\mathbf{S}), \quad \sum_{l}\left|I_{\mathbf{T}_{l}}\right| \leq C_{1} \mathcal{E}_{k}(\mathbf{S}, f)^{-2} .
$$

This lemma and its proof are well represented in the literature. For the multidimensional version, see Pramanik and Terwilleger [14].

To begin the decomposition, choose $m_{0}>0$ so that $\mathcal{E}_{k}\left(\mathbf{S}, f_{k}\right) \leq 2^{m_{0} n}$ for $k=1,2,3$. Using Lemma 2 , we can inductively construct a sequence of pairwise disjoint sets $\mathbf{S}_{m_{0}}, \mathbf{S}_{m_{0}-1}, \ldots$ such that

$$
\begin{aligned}
& \mathbf{S}=\bigcup_{m=-\infty}^{m_{0}} \mathbf{S}_{m}, \\
& \mathcal{E}_{k}\left(\mathbf{S}_{m}, f_{k}\right) \leq 2^{(m-1) n} \text { for all } m \leq m_{0} \text { and } k=1,2,3,
\end{aligned}
$$


(4.3) $\quad \mathbf{S}_{m}$ is a union of trees $\mathbf{T}_{l m}$ such that

$$
\sum_{l}\left|I_{\mathbf{T}_{l m}}\right| \leq C 2^{-2 m n} \quad \text { for all } m \leq m_{0}
$$

Here, $C$ is a constant depending only on dimension and the constant $C_{1}$ in Lemma 2. Thus we suppress its explicit value throughout the proof. Proofs of similar constructions can be found in Bilyk and Grafakos [1] or Pramanik and Terwilleger [14].

In Proposition 2, given a finite set $\mathbf{S}$ of tri-tiles, we are interested in those tri-tiles in the set

$$
\widetilde{\mathbf{S}}:=\left\{s \in \mathbf{S}: I_{s} \not \subset \Omega\right\} .
$$

Our restriction to $\widetilde{\mathbf{S}}$ is needed in the following lemma, which is a refined energy estimate.

Lemma 3. For $k=1,2$ and $f_{k}=\chi_{F_{k}}$,

$$
\mathcal{E}_{k}\left(\widetilde{\mathbf{S}}, f_{k}\right) \lesssim \min \left(\frac{\left|F_{k}\right|^{1 / 2}}{|E|},\left|F_{k}\right|^{-1 / 2}\right) .
$$

This lemma is crucial for obtaining the full range of exponents in our theorem. We are able to exploit the fact that we are working on a restricted class of tiles to obtain an improved estimate over (4.2). The proof of this lemma is quite technical and in the higher dimensional setting may be found in Grafakos, Tao, and Terwilleger [7].

Finally, before we commence our proof of the proposition, we need to estimate the operator over a single tree $\mathbf{T} \subseteq \mathbf{S}$. As noted earlier, any tree $\mathbf{T}$ can be written as the union of three trees each of type $j$ for at least two choices of $j$. Thus if we fix a $k=1,2,3$, we may assume $\mathbf{T}$ is a $j$-tree for $j \neq k$. In addition $\{s\}$ is a $k$-tree for all $k$, and so for all $s \in \mathbf{S}$,

$$
\left|I_{s}\right|^{-1 / 2}\left|\left\langle f_{k}, \phi_{s_{k}}\right\rangle\right| \leq \mathcal{E}_{k}\left(\mathbf{S}, f_{k}\right)\left\|f_{k}\right\|_{2} \text {. }
$$

Applying the above estimate and Cauchy-Schwarz, we see that

$$
\begin{aligned}
\left|\left\langle H_{\mathbf{T}}\left(f_{1}, f_{2}\right), f_{3}\right\rangle\right| & \leq \sum_{s \in \mathbf{T}}\left|I_{s}\right|^{-1 / 2}\left|\left\langle f_{k}, \phi_{s_{k}}\right\rangle\right| \prod_{j \neq k}\left|\left\langle f_{j}, \phi_{s_{j}}\right\rangle\right| \\
& \leq \mathcal{E}_{k}\left(\mathbf{S}, f_{k}\right)\left\|f_{k}\right\|_{2} \sum_{s \in \mathbf{T}} \prod_{j \neq k}\left|\left\langle f_{j}, \phi_{s_{j}}\right\rangle\right| \\
& \leq \mathcal{E}_{k}\left(\mathbf{S}, f_{k}\right)\left\|f_{k}\right\|_{2}\left|I_{\mathbf{T}}\right| \prod_{j \neq k}\left(\left|I_{\mathbf{T}}\right|^{-1} \sum_{s \in \mathbf{T}}\left|\left\langle f_{j}, \phi_{s_{j}}\right\rangle\right|^{2}\right)^{1 / 2} \\
& \leq\left|I_{\mathbf{T}}\right| \prod_{k=1}^{3} \mathcal{E}_{k}\left(\mathbf{S}, f_{k}\right)\left\|f_{k}\right\|_{2} .
\end{aligned}
$$


Now we have all the ingredients to deduce Proposition 2. Let $f_{1}=\chi_{F_{1}}$, $f_{2}=\chi_{F_{2}}$, and $f_{3}=\chi_{E^{\prime}}$ in the decomposition (4.1)-(4.3) of $\widetilde{\mathbf{S}}$ and in the estimate (4.5). Then we readily have

$$
\left|\left\langle H_{\widetilde{\mathbf{S}}}\left(\chi_{F_{1}}, \chi_{F_{2}}\right), \chi_{E^{\prime}}\right\rangle\right| \leq \sum_{m=-\infty}^{\infty} \sum_{l}\left|\left\langle H_{\mathbf{T}_{l m}}\left(\chi_{F_{1}}, \chi_{F_{2}}\right), \chi_{E^{\prime}}\right\rangle\right|
$$

$$
\begin{aligned}
\leq & \sum_{m=-\infty}^{\infty} \sum_{l}\left|I_{\mathbf{T}_{l m}}\right| \mathcal{E}_{1}\left(\mathbf{S}_{m}, \chi_{F_{1}}\right) \mathcal{E}_{2}\left(\mathbf{S}_{m}, \chi_{F_{2}}\right) \mathcal{E}_{3}\left(\mathbf{S}_{m}, \chi_{E^{\prime}}\right)\left|F_{1}\right|^{1 / 2}\left|F_{2}\right|^{1 / 2}|E|^{1 / 2} \\
\lesssim & \sum_{m=-\infty}^{\infty} 2^{-m n} \min \left(\frac{\left|F_{1}\right|^{1 / 2}}{|E|},\left|F_{1}\right|^{-1 / 2}, 2^{m n}\right) \min \left(\frac{\left|F_{2}\right|^{1 / 2}}{|E|},\left|F_{2}\right|^{-1 / 2}, 2^{m n}\right) \\
& \times\left|F_{1}\right|^{1 / 2}\left|F_{2}\right|^{1 / 2}|E|^{1 / 2}
\end{aligned}
$$

where the last inequality follows by applying (4.3) and (4.2) in addition to (4.4), which gives better estimates for the 1- and 2-energies when $m$ is large.

It remains to see that the sum gives the claimed estimate of Proposition 2. We consider several cases, but they all give geometric series plus a finite number of terms of logarithmic order.

CASE 1: $|E| \geq\left|F_{2}\right|,\left|F_{1}\right|$. If $\left|F_{2}\right| \geq\left|F_{1}\right|$, then the sum on the right hand side of (4.6) can be bounded by

$$
\begin{aligned}
&\left(\sum_{m=-\infty}^{\frac{1}{n} \log \frac{\left|F_{1}\right|^{1 / 2}}{|E|}} 2^{m n}+\sum_{m=\frac{1}{n} \log \frac{\left|F_{1}\right|^{1 / 2}}{|E|}}^{\frac{1}{n} \log \frac{\left|F_{2}\right|^{1 / 2}}{|E|}} \frac{\left|F_{1}\right|^{1 / 2}}{|E|}+\right.\left.\sum_{\begin{array}{c}
m=\frac{1}{n} \log \frac{\left|F_{2}\right|^{1 / 2}}{|E|} \\
\times
\end{array}}^{\infty} \frac{\left|F_{1}\right|^{1 / 2}\left|F_{2}\right|^{1 / 2}}{|E|^{2}}\right) \\
& \times\left|F_{1}\right|^{1 / 2}\left|F_{2}\right|^{1 / 2}|E|^{1 / 2} \\
& \lesssim\left|F_{1}\right|\left|F_{2}\right|^{1 / 2}|E|^{-1 / 2}\left(1+\log \frac{\left|F_{2}\right|}{\left|F_{1}\right|}\right)
\end{aligned}
$$

Reversing the roles of $F_{1}$ and $F_{2}$ gives a symmetric inequality, and so this case yields the bound

$$
\min \left(\left|F_{1}\right|^{1 / 2},\left|F_{2}\right|^{1 / 2}\right)\left|F_{1}\right|^{1 / 2}\left|F_{2}\right|^{1 / 2}|E|^{-1 / 2}\left(1+\left|\log \frac{\left|F_{2}\right|}{\left|F_{1}\right|}\right|\right) .
$$

This implies the statement of Proposition 2 since in this case $\left|F_{1}\right|\left|F_{2}\right||E|^{-1}$ $\leq|E|$.

CASE 2: $|E| \leq\left|F_{2}\right|,\left|F_{1}\right|$. This time we have $\left|F_{1}\right|\left|F_{2}\right||E|^{-1} \geq|E|$. Assuming $\left|F_{2}\right| \geq\left|F_{1}\right|$, (4.6) can be bounded by 


$$
\begin{array}{r}
\left(\sum_{m=-\infty}^{-\frac{1}{n} \log \left|F_{2}\right|^{1 / 2}} 2^{m n}+\sum_{m=-\frac{1}{n} \log \left|F_{2}\right|^{1 / 2}}^{-\frac{1}{n} \log \left|F_{1}\right|^{1 / 2}} \frac{1}{\left|F_{2}\right|^{1 / 2}}+\sum_{m=-\frac{1}{n} \log \left|F_{1}\right|^{1 / 2}}^{\infty} 2^{-m n} \frac{1}{\left|F_{1}\right|^{1 / 2}\left|F_{2}\right|^{1 / 2}}\right) \\
\times\left|F_{1}\right|^{1 / 2}\left|F_{2}\right|^{1 / 2}|E|^{1 / 2} \\
\lesssim\left|F_{1}\right||E|^{1 / 2}\left(1+\log \frac{\left|F_{1}\right|}{\left|F_{2}\right|}\right),
\end{array}
$$

and so by symmetry we have the bound

$$
\min \left(\left|F_{1}\right|^{1 / 2},\left|F_{2}\right|^{1 / 2}\right)|E|^{1 / 2}\left(1+\left|\log \frac{\left|F_{2}\right|}{\left|F_{1}\right|}\right|\right) .
$$

CASE 3: $|E|$ lies between $\left|F_{1}\right|$ and $\left|F_{2}\right|$ and $\left|F_{1}\right|\left|F_{2}\right||E|^{-1} \leq|E|$. Assuming $\left|F_{1}\right| \leq|E| \leq\left|F_{2}\right|$, (4.6) is estimated by

$$
\begin{array}{r}
\left(\sum_{m=-\infty}^{\frac{1}{n} \log \frac{\left|F_{1}\right|^{1 / 2}}{|E|} 2^{m n}+} \sum_{m=\frac{1}{n} \log \frac{\left|F_{1}\right|^{1 / 2}}{|E|}}^{-\frac{1}{n} \log \left|F_{2}\right|^{1 / 2}} \frac{\left|F_{1}\right|^{1 / 2}}{|E|}+\sum_{m=-\frac{1}{n} \log \left|F_{2}\right|^{1 / 2}}^{\infty} 2^{-m n} \frac{\left|F_{1}\right|^{1 / 2}}{\left|F_{2}\right|^{1 / 2}|E|}\right) \\
\times\left|F_{1}\right|^{1 / 2}\left|F_{2}\right|^{1 / 2}|E|^{1 / 2} \\
\lesssim\left|F_{1}\right|\left|F_{2}\right|^{1 / 2}|E|^{-1 / 2}\left(1+\log \frac{|E|^{2}}{\left|F_{1}\right|\left|F_{2}\right|}\right)
\end{array}
$$

So when $|E|$ lies between $\left|F_{1}\right|$ and $\left|F_{2}\right|$ and $\left|F_{1}\right|\left|F_{2}\right||E|^{-1} \leq|E|$, we obtain the bound

$$
\min \left(\left|F_{1}\right|^{1 / 2},\left|F_{2}\right|^{1 / 2}\right)\left|F_{1}\right|^{1 / 2}\left|F_{2}\right|^{1 / 2}|E|^{-1 / 2}\left(1+\log \frac{|E|^{2}}{\left|F_{1}\right|\left|F_{2}\right|}\right) .
$$

CASE 4: $|E|$ lies between $\left|F_{1}\right|$ and $\left|F_{2}\right|$ and $\left|F_{1}\right|\left|F_{2}\right||E|^{-1} \geq|E|$. Finally, if $\left|F_{1}\right| \leq|E| \leq\left|F_{2}\right|$ and $\left|F_{1}\right|\left|F_{2}\right||E|^{-1} \geq|E|$, we bound (4.6) by

$$
\begin{aligned}
& \left(^{-\frac{1}{n} \log \left|F_{2}\right|^{1 / 2}} \quad \frac{1}{n} \log \left|F_{1}\right|^{1 / 2} /|E|\right. \\
& \sum_{m=-\infty}^{n} 2^{m n}+\sum_{m=-\frac{1}{n} \log \left|F_{2}\right|^{1 / 2}}\left|F_{2}\right|^{-1 / 2} \\
& \left.+\sum_{m=\frac{1}{n} \log \frac{\left|F_{1}\right|^{1 / 2}}{|E|}}^{\infty} 2^{-m n}\left|F_{1}\right|^{1 / 2}\left|F_{2}\right|^{-1 / 2}|E|^{-1}\right) \\
& \times\left|F_{1}\right|^{1 / 2}\left|F_{2}\right|^{1 / 2}|E|^{1 / 2} \\
& \lesssim\left|F_{1}\right|^{1 / 2}|E|^{1 / 2}\left(1+\log \left|F_{1}\right|\left|F_{2}\right||E|^{-2}\right) .
\end{aligned}
$$

Thus symmetry in $F_{1}$ and $F_{2}$ yields the bound

$$
\min \left(\left|F_{1}\right|^{1 / 2},\left|F_{2}\right|^{1 / 2}\right)|E|^{1 / 2}\left(1+\left|\log \frac{\left|F_{1}\right|\left|F_{2}\right|}{|E|^{2}}\right|\right) \text {. }
$$

Our proof of Proposition 2 is now complete. 


\section{References}

[1] D. Bilyk and L. Grafakos, Distributional estimates for the bilinear Hilbert transform, J. Geom. Anal., to appear.

[2] - - - A new way of looking at distributional estimates; applications for the bilinear Hilbert transform, Collect. Math. 2006, special volume, 141-169.

[3] L. Carleson, On convergence and growth of partial sums of Fourier series, Acta Math. 116 (1966), 135-157.

[4] C. Fefferman, Pointwise convergence of Fourier series, Ann. of Math. (2) 98 (1973), $551-571$.

[5] L. Grafakos and X. C. Li, Uniform bounds for the bilinear Hilbert transforms. I, Ann. of Math. (2) 159 (2004), 889-933.

[6] L. Grafakos and T. Tao, Multilinear interpolation between adjoint operators, J. Funct. Anal. 199 (2003), 379-385.

[7] L. Grafakos, T. Tao, and E. Terwilleger, $L^{p}$ bounds for a maximal dyadic sum operator, Math. Z. 246 (2004), 321-337.

[8] R. A. Hunt, On the convergence of Fourier series, in: Orthogonal Expansions and their Continuous Analogues (Edwardsville, IL, 1967), Southern Illinois Univ. Press, Carbondale, IL, 1968, 235-255.

[9] M. T. Lacey, On the bilinear Hilbert transform, Doc. Math., 1998, Extra Vol. II, 647-656.

[10] M. Lacey and C. Thiele, $L^{p}$ estimates on the bilinear Hilbert transform for $2<p<\infty$, Ann. of Math. (2) 146 (1997), 693-724.

[11] —, - On Calderón's conjecture, Ann. of Math. (2) 149 (1999), 475-496.

[12] - - - A proof of boundedness of the Carleson operator, Math. Res. Lett. 7 (2000), 361-370.

[13] C. Muscalu, T. Tao, and C. Thiele, Multi-linear operators given by singular multipliers, J. Amer. Math. Soc. 15 (2002), 469-496.

[14] M. Pramanik and E. Terwilleger, $A$ weak $L^{2}$ estimate for a maximal dyadic sum operator on $\mathbb{R}^{n}$, Illinois J. Math. 47 (2003), 775-813.

Department of Mathematics, U-3009

University of Connecticut

Storrs, CT 06269, U.S.A.

E-mail: terwilleger@math.uconn.edu

Received July 11, 2006

Revised version January 9, 2007 